Supplement of

\title{
Ship-board determination of whole-rock (ultra-)trace element concentra- tions by laser ablation-inductively coupled plasma mass spectrometry anal- ysis of pressed powder pellets aboard the D/V Chikyu
}

Mathieu Rospabé et al.

Correspondence to: Mathieu Rospabé (mrospabe@jamstec.go.jp)

The copyright of individual parts of the supplement might differ from the article licence. 
Trace element concentrations in the four studied gabbros from the Oman Drilling Project CM cores (LA-ICP-MS D/V Chikyu and replicate analyses following Leg 3)

\begin{tabular}{|c|c|c|c|c|c|c|c|c|c|c|c|c|c|c|c|c|}
\hline & \multicolumn{4}{|c|}{ CM1A-6Z-2, $12-17 \mathrm{~cm}$} & \multicolumn{4}{|c|}{ CM1A-18Z-1, 26-33 cm } & \multicolumn{4}{|c|}{ CM1A-25Z-4, $50-59 \mathrm{~cm}$} & \multicolumn{4}{|c|}{ CM1A-41Z-2, 0-6 cm } \\
\hline & $\mathrm{RV}^{*}$ & Mean $(n=5)$ & SD & $\%$ RSD & $\mathrm{RV}^{*}$ & Mean $(n=5)$ & SD & $\%$ RSD & $\mathrm{RV}^{*}$ & Mean $(n=6)$ & SD & $\%$ RSD & $\mathrm{RV}^{*}$ & Mean $(n=6)$ & SD & $\%$ RSD \\
\hline Li & 1,75 & 1,49 & 0,215 & $14,5 \%$ & 1,65 & 1,59 & 0,132 & $8,4 \%$ & 0,669 & 0,628 & 0,0306 & $4,9 \%$ & 0,748 & 0,686 & 0,167 & $24,3 \%$ \\
\hline Sc & 44,5 & 39,7 & 2,57 & $6,5 \%$ & 14,5 & 15,7 & 1,65 & $10,5 \%$ & 40,9 & 40,8 & 2,78 & $6,8 \%$ & 51,0 & 43,9 & 3,17 & $7,2 \%$ \\
\hline $\mathrm{Ti}$ & 1202 & 1024 & 61,5 & $6,0 \%$ & 420 & 415 & 140 & $33,7 \%$ & 2834 & 2767 & 579 & $20,9 \%$ & 1648 & 1394 & 81,6 & $5,9 \%$ \\
\hline v & 112 & 117 & 9,20 & $7,9 \%$ & 46,2 & 48,6 & 18,0 & $36,9 \%$ & 171 & 198 & 15,8 & $8,0 \%$ & 158 & 173 & 12,3 & $7,1 \%$ \\
\hline $\mathrm{Cr}$ & 1122 & 1085 & 113 & $10,4 \%$ & 733 & 745 & 233 & $31,2 \%$ & 565 & 522 & 49,0 & $9,4 \%$ & 391 & 386 & 38,3 & $9,9 \%$ \\
\hline $\mathrm{Mn}$ & n.a. ${ }^{* *}$ & 547 & 47,4 & $8,7 \%$ & n.a. ${ }^{* *}$ & 265 & 39,8 & $15,0 \%$ & n.a. ${ }^{* *}$ & 764 & 65,3 & $8,5 \%$ & n.a. ${ }^{* *}$ & 976 & 51,0 & $5,2 \%$ \\
\hline co & 30,9 & 31,0 & 3,63 & $11,7 \%$ & 12,9 & 13,2 & 1,53 & $11,7 \%$ & 41,5 & 42,9 & 3,72 & $8,7 \%$ & 41,9 & 46,7 & 5,70 & $12,2 \%$ \\
\hline $\mathrm{Ni}$ & 209 & 201 & 25,4 & $12,6 \%$ & 95,9 & 100 & 14,2 & $14,2 \%$ & 160 & 170 & 16,4 & $9,7 \%$ & 128 & 146 & 15,1 & $10,4 \%$ \\
\hline $\mathrm{Cu}$ & 151 & 151 & 22,0 & $14,6 \%$ & 66,7 & 55,4 & 5,96 & $10,8 \%$ & 97,5 & 101 & 10,9 & $10,8 \%$ & 121 & 136 & 29,6 & $21,8 \%$ \\
\hline $\mathrm{Zn}$ & 18,9 & 18,0 & 2,88 & $15,9 \%$ & 7,48 & 6,87 & 1,59 & $23,2 \%$ & 17,1 & 13,7 & 3,22 & $23,5 \%$ & 24,8 & 31,3 & 6,50 & $20,8 \%$ \\
\hline Ga & n.a. ${ }^{* *}$ & 9,90 & 1,16 & $11,7 \%$ & n.a. ${ }^{* *}$ & 8,99 & 0,745 & $8,3 \%$ & n.a. ${ }^{* *}$ & 7,19 & 0,462 & $6,4 \%$ & n.a. ${ }^{* *}$ & 9,85 & 0,727 & $7,4 \%$ \\
\hline $\mathrm{Rb}$ & 0,685 & 0,628 & 0,103 & $16,4 \%$ & 1,02 & 0,974 & 0,0925 & $9,5 \%$ & 0,137 & 0,130 & 0,0236 & $18,2 \%$ & 0,0570 & 0,0555 & 0,0170 & $30,6 \%$ \\
\hline $\mathrm{Sr}$ & 174 & 158 & 15,7 & $9,9 \%$ & 174 & 189 & 21,6 & $11,5 \%$ & 189 & 221 & 10,4 & $4,7 \%$ & 137 & 138 & 8,12 & $5,9 \%$ \\
\hline $\mathrm{Y}$ & 4,24 & 4,12 & 0,299 & $7,2 \%$ & 1,07 & 1,19 & 0,377 & $31,6 \%$ & 7,46 & 8,69 & 1,23 & $14,2 \%$ & 6,42 & 6,12 & 0,480 & $7,8 \%$ \\
\hline $\mathrm{zr}$ & 2,93 & 2,55 & 0,173 & $6,8 \%$ & 1,27 & 1,06 & 0,144 & $13,6 \%$ & 5,80 & 6,13 & 0,902 & $14,7 \%$ & 3,12 & 2,68 & 0,196 & $7,3 \%$ \\
\hline $\mathrm{Nb}$ & 0,0280 & 0,0064 & 0,0012 & $19,6 \%$ & 0,0230 & 0,009 & 0,0046 & $50,5 \%$ & 0,0700 & 0,0680 & 0,0116 & $17,1 \%$ & 0,0150 & 0,0068 & 0,0016 & $22,9 \%$ \\
\hline Cs & 0,286 & 0,240 & 0,0537 & $22,4 \%$ & 0,813 & 0,756 & 0,0604 & $8,0 \%$ & 0,0540 & 0,0484 & 0,0243 & $50,2 \%$ & 0,0220 & 0,0331 & 0,0021 & $6,3 \%$ \\
\hline $\mathrm{Ba}$ & 8,54 & 8,08 & 0,751 & $9,3 \%$ & 5,31 & 5,78 & 0,370 & $6,4 \%$ & 2,09 & 2,40 & 0,0882 & $3,7 \%$ & 2,54 & 2,91 & 0,109 & $3,8 \%$ \\
\hline La & 0,149 & 0,135 & 0,0103 & $7,6 \%$ & 0,0840 & 0,0877 & 0,0126 & $14,4 \%$ & 0,346 & 0,322 & 0,0321 & $10,0 \%$ & 0,162 & 0,154 & 0,0098 & $6,3 \%$ \\
\hline $\mathrm{Ce}$ & 0,513 & 0,517 & 0,0371 & $7,2 \%$ & 0,244 & 0,261 & 0,0268 & $10,3 \%$ & 1,06 & 1,14 & 0,147 & $12,9 \%$ & 0,591 & 0,667 & 0,0367 & $5,5 \%$ \\
\hline $\mathrm{Pr}$ & 0,117 & 0,112 & 0,0063 & $5,6 \%$ & 0,0400 & 0,0425 & 0,0086 & $20,1 \%$ & 0,221 & 0,239 & 0,0300 & $12,6 \%$ & 0,143 & 0,143 & 0,0100 & $7,0 \%$ \\
\hline $\mathrm{Nd}$ & 0,766 & 0,730 & 0,0356 & $4,9 \%$ & 0,242 & 0,261 & 0,0548 & $21,0 \%$ & 1,41 & 1,54 & 0,204 & $13,2 \%$ & 0,996 & 0,995 & 0,0429 & $4,3 \%$ \\
\hline $\mathrm{Sm}$ & 0,370 & 0,355 & 0,0164 & $4,6 \%$ & 0,108 & 0,121 & 0,0255 & $21,1 \%$ & 0,645 & 0,701 & 0,116 & $16,5 \%$ & 0,527 & 0,511 & 0,0501 & $9,8 \%$ \\
\hline $\mathrm{Eu}$ & 0,249 & 0,234 & 0,0074 & $3,2 \%$ & 0,117 & 0,119 & 0,0084 & $7,1 \%$ & 0,355 & 0,386 & 0,0476 & $12,3 \%$ & 0,326 & 0,318 & 0,0200 & $6,3 \%$ \\
\hline Gd & 0,612 & 0,597 & 0,0469 & $7,9 \%$ & 0,164 & 0,159 & 0,0410 & $25,7 \%$ & 1,03 & 1,15 & 0,182 & $15,8 \%$ & 0,892 & 0,824 & 0,0695 & $8,4 \%$ \\
\hline $\mathrm{Tb}$ & 0,116 & 0,105 & 0,0064 & $6,1 \%$ & 0,0290 & 0,0315 & 0,0097 & $30,9 \%$ & 0,193 & 0,214 & 0,0367 & $17,1 \%$ & 0,171 & 0,145 & 0,0176 & $12,1 \%$ \\
\hline Dy & 0,761 & 0,735 & 0,0528 & $7,2 \%$ & 0,188 & 0,213 & 0,0719 & $33,7 \%$ & 1,30 & 1,49 & 0,208 & $13,9 \%$ & 1,14 & 1,05 & 0,111 & $10,6 \%$ \\
\hline Ho & 0,161 & 0,156 & 0,0058 & $3,7 \%$ & 0,0390 & 0,0439 & 0,0145 & $32,9 \%$ & 0,278 & 0,325 & 0,0465 & $14,3 \%$ & 0,244 & 0,225 & 0,0184 & $8,2 \%$ \\
\hline $\mathrm{Er}$ & 0,446 & 0,419 & 0,0313 & $7,5 \%$ & 0,108 & 0,123 & 0,0447 & $36,3 \%$ & 0,795 & 0,906 & 0,114 & $12,6 \%$ & 0,679 & 0,633 & 0,0462 & $7,3 \%$ \\
\hline $\mathrm{Tm}$ & 0,0620 & 0,0591 & 0,0020 & $3,4 \%$ & 0,0150 & 0,0179 & 0,0051 & $28,3 \%$ & 0,118 & 0,137 & 0,0205 & $15,0 \%$ & 0,0940 & 0,0926 & 0,0088 & $9,5 \%$ \\
\hline $\mathrm{Yb}$ & 0,386 & 0,360 & 0,0317 & $8,8 \%$ & 0,0940 & 0,111 & 0,0281 & $25,3 \%$ & 0,744 & 0,822 & 0,0905 & $11,0 \%$ & 0,592 & 0,530 & 0,0684 & $12,9 \%$ \\
\hline Lu & 0,0540 & 0,0508 & 0,0045 & $8,9 \%$ & 0,0130 & 0,0135 & 0,0029 & $21,2 \%$ & 0,112 & 0,121 & 0,0117 & $9,6 \%$ & 0,0860 & 0,0776 & 0,0058 & $7,5 \%$ \\
\hline $\mathrm{Hf}$ & 0,147 & 0,135 & 0,0043 & $3,2 \%$ & 0,0490 & 0,0439 & 0,0032 & $7,2 \%$ & 0,250 & 0,282 & 0,0469 & $16,7 \%$ & 0,174 & 0,158 & 0,0127 & $8,0 \%$ \\
\hline $\mathrm{Ta}$ & 0,0160 & 0,0014 & 0,0006 & $47,0 \%$ & 0,0110 & 0,0006 & 0,0004 & $66,0 \%$ & 0,0170 & 0,0051 & 0,0007 & $13,7 \%$ & 0,0080 & 0,0021 & 0,0020 & $97,1 \%$ \\
\hline $\mathrm{Pb}$ & 0,161 & 0,100 & 0,0209 & $20,8 \%$ & 0,0990 & 0,124 & 0,0540 & $43,7 \%$ & 0,0820 & 0,0630 & 0,0159 & $25,3 \%$ & 0,116 & 0,127 & 0,0465 & $36,6 \%$ \\
\hline Th & 0,0020 & 0,0007 & 0,0004 & $62,4 \%$ & 0,0020 & 0,0013 & 0,0009 & $65,0 \%$ & 0,0090 & 0,0086 & 0,0020 & $23,2 \%$ & 0,0020 & 0,0009 & 0,0005 & $55,8 \%$ \\
\hline U & 0,0010 & 0,0019 & 0,0012 & $65,6 \%$ & 0,0010 & 0,0026 & 0,0013 & $50,4 \%$ & 0,0040 & 0,0029 & 0,0012 & $42,8 \%$ & 0,0010 & 0,0010 & 0,0007 & $75,1 \%$ \\
\hline
\end{tabular}

* replicate values acquired by solution analyses by ICP-MS at the University of Southampton

** not analysed 
Trace element concentrations in the three studied dunites from the Oman Drilling Project CM cores (LA-ICP-MS D/V Chikyu and replicate analyses following Leg 3)

\begin{tabular}{|c|c|c|c|c|c|c|c|c|c|c|c|c|}
\hline & \multicolumn{4}{|c|}{ CM1A-51Z-1, 31-39 cm } & \multicolumn{4}{|c|}{ CM1A-58Z-2, 1-6 cm } & \multicolumn{4}{|c|}{ CM1A-75Z-3, $66-73 \mathrm{~cm}$} \\
\hline & $\mathrm{RV}^{*}$ & Mean $(n=6)$ & SD & $\%$ RSD & $\mathrm{RV}^{*}$ & Mean $(n=6)$ & SD & $\%$ RSD & $\mathrm{RV}^{*}$ & Mean $(n=5)$ & SD & $\%$ RSD \\
\hline $\mathrm{Y}$ & 0,118 & 0,105 & 0,0070 & $6,6 \%$ & 0,111 & 0,162 & 0,0080 & $5,0 \%$ & 0,0571 & 0,0853 & 0,0129 & $15,1 \%$ \\
\hline $\mathrm{Zr}$ & 0,147 & 0,0936 & 0,0082 & $8,7 \%$ & 0,121 & 0,0562 & 0,0033 & $5,8 \%$ & 0,0598 & 0,0501 & 0,0091 & $18,2 \%$ \\
\hline $\mathrm{Nb}$ & 0,0103 & 0,0014 & 0,0009 & $68,9 \%$ & 0,0159 & 0,0037 & 0,0022 & $60,7 \%$ & 0,0152 & 0,0073 & 0,0048 & $66,3 \%$ \\
\hline La & 0,0041 & 0,0024 & 0,0005 & $20,1 \%$ & 0,0113 & 0,0118 & 0,0038 & $32,2 \%$ & 0,0198 & 0,0127 & 0,0092 & $72,5 \%$ \\
\hline $\mathrm{Ce}$ & 0,0113 & 0,0083 & 0,0012 & $13,9 \%$ & 0,0254 & n.d. ${ }^{* *}$ & & & 0,0486 & 0,0214 & 0,0097 & $45,2 \%$ \\
\hline $\operatorname{Pr}$ & 0,0019 & 0,0014 & 0,0003 & $18,5 \%$ & 0,0034 & 0,0032 & 0,0006 & $17,9 \%$ & 0,0016 & 0,0017 & 0,0011 & $64,2 \%$ \\
\hline $\mathrm{Nd}$ & 0,0113 & 0,0085 & 0,0015 & $17,7 \%$ & 0,0148 & 0,0162 & 0,0031 & $19,4 \%$ & 0,0062 & 0,0070 & 0,0041 & $58,9 \%$ \\
\hline $\mathrm{Sm}$ & 0,0047 & 0,0036 & 0,0028 & $76,4 \%$ & 0,0043 & 0,0056 & 0,0016 & $29,2 \%$ & 0,0023 & 0,0023 & 0,0020 & $90,0 \%$ \\
\hline Eu & 0,0079 & 0,0072 & 0,0010 & $13,9 \%$ & 0,0086 & 0,0116 & 0,0018 & $15,8 \%$ & 0,0007 & 0,0018 & 0,0007 & $36,2 \%$ \\
\hline$G d$ & 0,0080 & 0,0080 & 0,0021 & $25,7 \%$ & 0,0064 & 0,0104 & 0,0019 & $18,2 \%$ & 0,0036 & 0,0039 & 0,0012 & $30,8 \%$ \\
\hline $\mathrm{Tb}$ & 0,0018 & 0,0013 & 0,0002 & $15,5 \%$ & 0,0015 & 0,0021 & 0,0007 & $34,0 \%$ & 0,0008 & 0,0011 & 0,0005 & $45,8 \%$ \\
\hline Dy & 0,0146 & 0,0111 & 0,0020 & $18,5 \%$ & 0,0131 & 0,0194 & 0,0025 & $12,8 \%$ & 0,0069 & 0,0090 & 0,0028 & $31,7 \%$ \\
\hline $\mathrm{Ho}$ & 0,0041 & 0,0033 & 0,0005 & $14,6 \%$ & 0,0041 & 0,0060 & 0,0011 & $18,8 \%$ & 0,0022 & 0,0027 & 0,0005 & $18,2 \%$ \\
\hline $\mathrm{Er}$ & 0,0155 & 0,0142 & 0,0018 & $12,9 \%$ & 0,0164 & 0,0214 & 0,0027 & $12,7 \%$ & 0,0087 & 0,0118 & 0,0023 & $19,5 \%$ \\
\hline $\mathrm{Yb}$ & 0,0267 & 0,0238 & 0,0028 & $11,8 \%$ & 0,0261 & 0,0423 & 0,0045 & $10,6 \%$ & 0,0187 & 0,0256 & 0,0054 & $21,0 \%$ \\
\hline Lu & 0,0055 & 0,0045 & 0,0006 & $12,4 \%$ & 0,0047 & 0,0078 & 0,0010 & $13,1 \%$ & 0,0041 & 0,0054 & 0,0013 & $24,7 \%$ \\
\hline $\mathrm{Hf}$ & 0,0046 & 0,0025 & 0,0013 & $54,0 \%$ & 0,0016 & 0,0025 & 0,0011 & $44,5 \%$ & 0,0020 & 0,0028 & 0,0008 & $27,9 \%$ \\
\hline $\mathrm{Ta}$ & n.d. ${ }^{* *}$ & 0,0008 & 0,0008 & $110,7 \%$ & 0,0027 & 0,0005 & 0,0002 & $41,6 \%$ & n.d. ${ }^{* *}$ & 0,0002 & 0,0001 & $34,8 \%$ \\
\hline $\mathrm{Pb}$ & 0,0168 & 0,0300 & 0,0130 & $43,4 \%$ & 0,0165 & 0,0392 & 0,0258 & $65,9 \%$ & 0,0089 & 0,0163 & 0,0070 & $42,9 \%$ \\
\hline Th & 0,0007 & 0,0004 & 0,0002 & $50,1 \%$ & 0,0004 & 0,0003 & 0,0002 & $82,3 \%$ & 0,0009 & 0,0005 & 0,0001 & $10,1 \%$ \\
\hline U & 0,0004 & 0,0005 & 0,0001 & $14,4 \%$ & 0,0002 & 0,0018 & 0,0005 & $29,6 \%$ & 0,0006 & 0,0016 & 0,0004 & $28,0 \%$ \\
\hline
\end{tabular}

* replicate values acquired by solution analyses by ICP-MS at the at the Institute of Earth Science, Academia Sinica at Taipei (Taiwan) and the Géosciences Environnement Toulouse laboratory, Université Toulouse III - Paul Sabatier (France); these data and concentrations for other trace elements are proposed in Kourim et al., submitted

** not determined

*** not analysed (Tm used as internal standard, see Barrat et al., 1996 and Rospabé et al., 2018a) 